\title{
Interference Avoidance Mechanisms in Bluetooth - A Comparative Study
}

\author{
Sughasiny M \\ Research Scholar, Research and Development \\ Centre \\ Bharathiyar University, Coimbatore-641 046, \\ TamilNadu, India
}

\author{
Dhanapal R \\ Professor and Head, Research Department of \\ Computer Applications \\ Easwari Engineering College, Chennai-600 \\ 089, TamilNadu, India
}

\begin{abstract}
Bluetooth is a short range operating technology that helps in the exchange of data. Faster and efficient communications using a Bluetooth device is mandatory, since it has resource limitations. Service discovery delays pose a major problem when considering the Bluetooth devices. Our paper provides a comparative study that discusses efficient mechanisms to reduce these delays by providing a better service discovery process. Further, we also discuss mechanisms that help improve the power consumption.
\end{abstract}

\section{KEYWORDS}

Service discovery; Bluetooth; Adaptive frequency hopping; Max-Min D Clustering

\section{INTRODUCTION}

Bluetooth [17] is a short-range wireless technology that allows devices to exchange data and voice in real-time. The Bluetooth Special Interest Group (SIG)[12] is responsible for developing specifications of this technology. Many mechanisms were proposed for efficient service discovery and device discovery. Key challenges in wireless mobile ad hoc networks are computational resource constraints, power limitations, and efficient service discovery techniques[13]. The short range radio network technology Bluetooth suffers from long service discovery delays and high power consumption due to necessary connection establishment between discovering and discovered entity.

Bluetooth Service Discovery Protocol (SDP)[12] enables a client application on a device to discover information about services on other Bluetooth devices. Every service is represented by a profile, identified by a 128-bit Universally Unique Identifier (UUID). A match occurs on a peer device if and only if at least one UUID specified by the client is contained in one or more of its service records. Efficient service discovery helps in efficient and longer usage of the network. Further, interference in Bluetooth frequencies poses a great threat in maintaining an efficient transfer. These interferences come from microwave ovens, other wireless devices etc. Hence the selection of frequencies should be in such a manner that reduces the amount of interference in a current channel.

Many academic researchers and practitioners in the commercial sectors have worked on a number of research projects to tackle the coexistence problem $[18,19,20,21]$. The significance of this interference problem is further recognized with the establishment of the Coexistence Task Group 2, which is set up by and coordinated from Bluetooth SIG (Special Interest Group) and IEEE 802.15 to fight against this problem [22]. Previously proposed solutions can be broadly divided into two categories: Collaborative and Noncollaborative. Collaborative mechanisms [18] require that Bluetooth and IEEE $802.11 \mathrm{~b}$ transceivers communicate with each other about their traffic to avoid interference. Noncollaborative mechanisms [23] do not involve such communications but the transceivers sense the existence of other type of wireless transmissions by estimating the channel conditions frequently[5].

A technique called frequency hopping helps to tackle this issue efficiently. The method of frequency hopping analyzes a frequency and if it contains interferences, then that particular frequency is omitted. But this technique proves to be costly when practically applying it on a real time environment. In contrast to traditional frequency hopping techniques, Adaptive Frequency Hopping (AFH) is a low cost and low power solution to avoid interference dynamically.

\section{BLUETOOTH}

Bluetooth is a short range wireless system operating in 2.4 $\mathrm{GHz}$ industry, society and medical (ISM) unlicensed band, where a number of radio systems share the same resource. The Bluetooth rapidly hops over all the 79 channels to mitigate interferences using a pseudo- random frequency hopping $(\mathrm{FH})$ technique [1]. It first generate a pseudo-random hop sequence

based on its master clock and address, and then maps it into a desired hopping frequency so that each packet is transmitted through a different channel. However, recent works have shown that the performance of the Bluetooth system using pseudorandom FH technique can be heavily degraded due to unavoidable co-existing interferences (e.g., interferences caused by collocated Bluetooth devices or IEEE 802.11x based wireless local area network (WLAN)) in the unlicensed band [2-6].

To alleviate this problem, a number of non-collaborative schemes working regardless of any other systems have been considered [7-12]. Most of them consider the use of two fundamental processes; channel classification and adaptive control action. Channel classification estimates channel conditions to detect the presence of any interference source nearby. Adaptive control action mitigates interference sources by exploiting the channel classification information. 


\section{COMPARISON STUDY}

\subsection{Clustering}

Clustering[1] is one of the most important research topics in both machine learning and data-mining communities. Clustering refers to partitioning the given data into groups. Every group contains objects that are similar in nature. Hence clustering helps in finding patterns in a group. This is an unsupervised learning technique, hence user interference is found to be minimal when performing the process of clustering. Normal clustering algorithms such as the k-means clustering algorithm performs the process of a simple logical grouping of nodes. Communications are usually carried out between the individual nodes.

In the mobility-based d-hop (MobDHop)[6] clustering algorithm, clusters of variable diameters are created based on the mobility patterns. Unlike existing clustering algorithms, the diameter of clusters is not restricted by any preset value. Instead, the diameter of clusters is flexible and determined by the stability of clusters. Nodes which have similar moving patterns are grouped into one cluster in order to achieve maximum cluster stability. Even though nodes belong to the same cluster, data transfer is performed by all the nodes. While in the Max-Min D-Clustering [24], every cluster contains a cluster head, and communications to the cluster can be carried out with that node alone. This helps reduce the amount of traffic inside a cluster. The cluster head receives the data and transfers it to the corresponding node.

\subsection{Adaptive Frequency Hopping}

Careful examination of AFH algorithms reveals that they belong to two classes; the first relies on reducing the cardinality of the hop-set, while the second approach relies on probabilistic channel visiting. In addition, these algorithms are optimal either in the presence of static sources of interference (SI) or in the presence of dynamic sources of interference (DI); some AFH algorithms go one step further and mitigate the effect of both SI and DI. Figure 1, illustrates the classification tree of AFH algorithms.

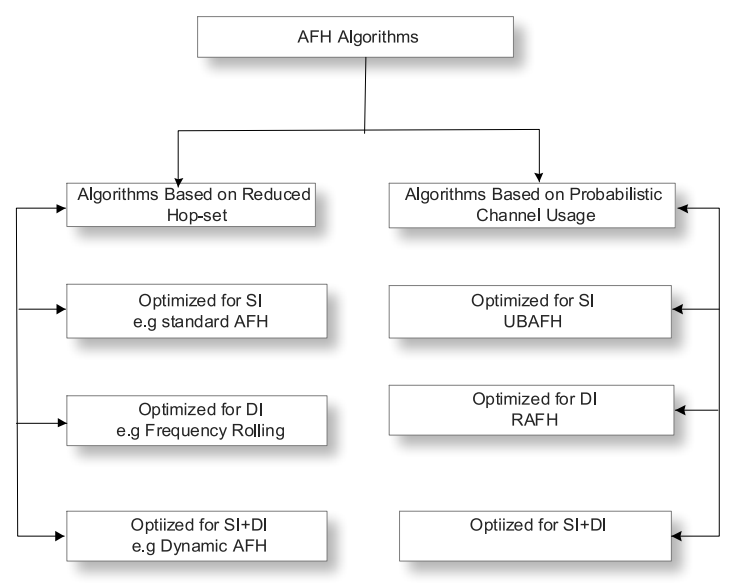

Fig. 1. Taxonomy of AFH techniques

Algorithms Based on Reduced hop-sets avoid bad channels completely; as a result the hop-set consists only of a small number of channels from the available spectrum. AFH is one of the most important non-collaborative coexistence mechanisms[8]. AFH generally consists of four phases. Device identification process is mainly used for backward compatibility and checking if a device can work in AFH mode. Channel classification is the process used for distinguishing the channel quality. There are a variety of implementations and this process is very important because the partition sequence generated by AFH kernel is based on the result of this part. Classification information exchange is the protocol for master and slave to exchange the results of their measurement. AFH kernel is the specific algorithm to choose the hop frequency. The goal of the algorithm is to avoid as many bad channels as possible. Standard Adaptive Frequency Hopping (AFH) [31] actively modifies the hopping sequence to use good channels and avoid interference. It is an effective measure in mitigating the interference resulting from frequency static devices such as IEEE 802.11b.

The standard AFH consists of three distinct components shown in Figure 19; the first component of the AFH mechanism is the selection box, which generates the hopping sequence defined in the IEEE Std 802.15.1-2002 [32]

The second component is the partition sequence generator, which imposes a structure on the original hopping sequence. It divides the set of "bad" channels $(S B)$, into a set of "bad" channels that are to be kept in the hopping sequence $(S B K)$, and into a set of "bad" channels that are to be removed from the hopping sequence $(S B R)$.

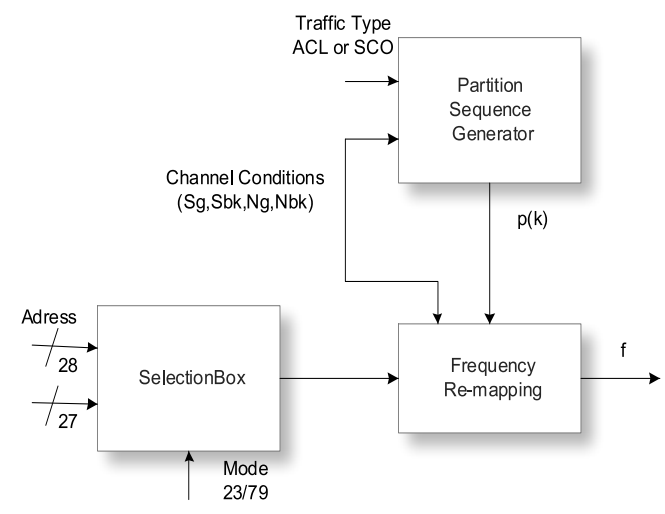

Fig. 2. AFH Mechanism

The set $S B K$ is needed in case the size of the set of "good" channels $(S G)$ is less than the minimum number of hopping channels allowed (Nmin). The size of each partition is given by the following two equation:

$$
\left\{\begin{array}{l}
N_{B K}=\max \left(0, N_{\min }-N_{G}\right) \\
N_{B R}=N_{B}-N_{B K}
\end{array}\right\}
$$

The partition sequence generates a flag $p(k)$ at each time slot $k$ to indicate if bad channels can be used or not; $p(k)=1$ when $N G<N$ min otherwise $p(k)=0$. The third component of the $\mathrm{AFH}$ mechanism is the frequency remapping function; it compares the hop frequency generated by the pseudo-random hop selection scheme against the two set of good and bad channels. If the channel belongs to the "good" channel list, it will just be used normally without any special action. On the other hand, if the frequency assigned by the original scheme is included in the "bad" channel list, a remapping function is invoked to substitute the "bad" channel according to the flowchart in Figure 3. 


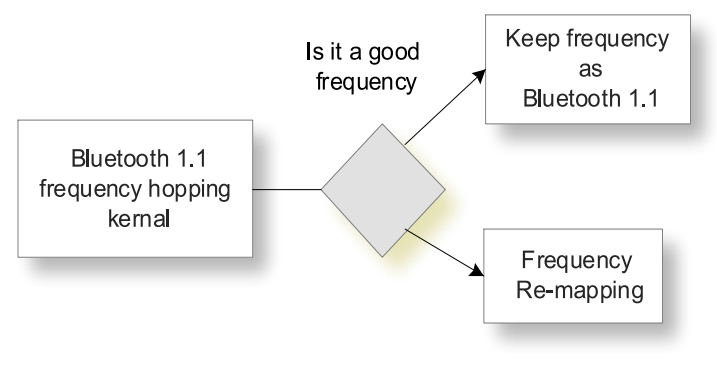

Fig. 3. AFH Frequency Decision Flowchart

The remapping function uses $p(k)$ to check if the number of "good" channels is greater than Nmin; if this is the case then all "bad" channels are remapped to "good" channels; otherwise some of the "bad" channels are still being used in order to conform to the regulation 17.

The Standard Adaptive Frequency Hopping periodically maintains the hopset to handle changing channel conditions. However it is difficult to decide on the appropriate period $T$ after which bad channels are introduced again in the hop-set.

Previous results have not considered spectrum characteristics of error sources in the channel classification and adaptive control action, i.e., wide and static transmission characteristic of WLAN, narrow and non-static transmission characteristic of interference from other Bluetooth and characteristic of channel noise that yields uniform error over the whole set of frequencies.

We have found that consideration for the spectrum characteristics of error sources can provide the potential performance improvement, minimizing the power consumption. In this paper, we propose a new AFH scheme that sequentially mitigate error sources exploiting the spectrum characteristic of the interference sources and channel noise.

MEHTA (MAC Enhanced Temporal Algorithm) proposed in [25], traffic information is exchanged between the Bluetooth and IEEE $802.11 \mathrm{~b}$ firmware to calculate the accurate timings at the MAC layer, avoiding interference by proper synchronization. Technically, it is an interference avoidance scheme in the time domain. With these schemes, modifications of the firmware and/or the hardware of the Bluetooth and IEEE $802.11 \mathrm{~b}$ transceivers are required. While the approach described in [26] \& [27] do not require changes in the firmware components. to provide high bandwidth stability at high data rates and lower drop rates for both interfaces under interference.

SAFH[7] assigns usage probabilities to all channels based on an exponential smoothing filter for frame error rates to estimate and predict the channel conditions. The application layer can adapt SAFH by parameter settings in a cross-layer approach. SAFH achieves low average frame error rate and responds fast to changing channel conditions if required from the application.

In [28] a partitioning of the original Bluetooth hop band is proposed to reduce the collisions between piconets. The described algorithm Orthogonal Hop Set Partitioning (OHSP) suggests five orthogonal sub-hopsets. Each master chooses randomly and independently one of these sub-hopsets. For best case combination of sub-hopsets the throughput could be improved by $10 \%$, but in worst case combination the algorithm shows even degradation. Therefore on average there is only a marginal improvement. Popkovski et al. propose a dynamic adaptive frequency hopping (DAFH) algorithm in [33]. According to the observed packet errors each piconet adapts its hopset to a subset of good frequency channels to minimize the interference. The drawback of OHSP and DAFH is that the hopset size is reduced and therefore the frequency diversity is decreased. This results in longer channel occupancy and therefore higher probability of collisions with concurrent radio links degrading their operation. Stabellini et al. propose a so-called Utility Based Adaptive Frequency Hopping (UBAFH) [29], [30]. UBAFH maps the observed packet error rate (PER) to a probability density function that defines the usage probability of each channel. In [29] the algorithm is evaluated for frequency selective fading channels and in [30] it is additionally evaluated under frequencydynamic interference.

Interference Source Oriented Adaptive Frequency Hopping (ISOAFH) approach based on a cross-layer design, in which the baseband layer of Bluetooth considers not only the instantaneous channels condition but also the physical layer transmission characteristics of potential interference sources in determining the hop sequence. The advantage of our approach when opposed to this approach is that Moreover we minimize the risk of incorrect classification due to instantaneous disturbances e.g. other frequency hoppers.

A collision occurs when the desired signal overlaps in time and in frequency with an interfering signal, therefore:

$$
P(C)=P(\text { overlap in time }) * P(\text { overlap in frequency })
$$

$\{9\}$ Interference Source Oriented AFH (ISOAFH) analyzing the interference source's (i.e., WLAN technologies) transmission characteristics. In particular, we stress on the radio transmission characteristics of IEEE 802.11 b. An IEEE $802.11 \mathrm{~b}$ system spreads the energy of the transmission signal on the spectrum over a chosen channel of bandwidth $22 \mathrm{MHz}$. However, the channel allocation is overlapping in nature with each channel separated by $5 \mathrm{MHz}$. The channels need to be separated by at least five channels to achieve zero overlap.

To derive $P$ (overlap in frequency), we recall that WLAN (802.11b) consists of three non overlapping channels with a bandwidth of $22 \mathrm{MHZ}$ each; in this topology only one channel is occupied by the WLAN as a result,

$$
P(\text { overlap in frequency })=\sum_{i=c}^{i=c+21} P_{i}
$$

Where $c$ is the first channel occupied by the WLAN, and $P i$ is the probability that $\mathrm{AFH}$ is using channel $i$.

We start the analysis for a $100 \%$ traffic load21 and extend the result to accommodate different duty cycles; Let TSAFH, TW and TBoff be the time of SAFH packet, the time of WLAN packet and the back-off time of the WLAN, respectively.

- When $T_{\text {SAFH }} \geq T_{\text {Boff }}$, there would be always an overlap in time,

and therefore $P(C)=\sum_{\substack{i=c+21 \\ i=c}} P_{i}$ 
- When SAFH transmission time is smaller than the WLAN backoff time i.e.

$T_{\text {SAFH }}<T_{\text {Boff }}$; there would not be overlap in time, if the SAFH packet hops during the back-off period.

- $\quad$ Let $\tilde{M}$ be the number of times packets overlap in time.

$M$ assumes two values:

$$
\left\{\begin{array}{c}
\tilde{M}=1 \text { with probability } \frac{T_{W}+T_{S A F H}}{T_{W I}} \\
\tilde{M}=0 \text { with probability } \frac{T_{B o f f}-T_{S A F H}}{T_{W I}}
\end{array}\right\}
$$

Where $T_{W I}=T_{w}+T_{B o f f}$ is the inter arrival time of WLAN packets. Using the theorem of total probability

$$
\begin{gathered}
P(C)=\sum\left[P(M=m)\left(1-(1-p)^{m}\right)\right] \Rightarrow \\
\left\{\begin{array}{c}
P(C)=\frac{T_{W}+T_{S A F H}}{T_{W I}}\left(1-\left(1-\sum_{i=c}^{i=c+21} P_{i}\right)^{1}\right)+\frac{T_{B o f f}-T_{S A F H}}{T_{W I}}(1-1) \\
=\frac{T_{W}+T_{S A F H}}{T_{W I}}\left(\sum_{i=c}^{i=c+21} P_{i}\right.
\end{array}\right\}
\end{gathered}
$$

For arbitrary traffic load $P_{L} \leq 100 \%$, we multiply the results obtained (28) by $\mathrm{P}_{\mathrm{L}}$. Here's the final result for the probability of collision of SAFH interfered by WLAN:

$$
\left\{\begin{array}{l}
P(C)=P_{L}\left(\sum_{i=c}^{i=c+21} P_{i}\right) \text { when } T_{S A F H} \geq T_{B o f f} \\
P(C)=P_{L}\left(\frac{T_{W}+T_{S A F H}}{T_{W I}}\right)\left(\sum_{i=c}^{i=c+21} P_{i}\right) \text { when } T_{S A F H}<T_{B o f f}
\end{array}\right\}
$$

In our proposed channel classification method, we do not intend to find individual "bad" channels. Instead, we try to locate the carrier(s) of IEEE $802.11 \mathrm{~b}$ interference source(s) and then attempt to avoid hopping on all the affected Bluetooth channels.

Users are allocated carriers that have the highest SNR of available system carriers[10]. This process is updated regularly to track channel fading. Adaptive frequency hopping greatly reduces frequency selective fading, improves interference rejection, and consequently improves the received SNR. Additionally Doppler spread is minimized due to the avoidance of nulls in the spectrum. These effects improve the system capacity and user access reliability. Improvements in system capacity outweigh the overhead required for implementation of adaptive frequency hopping for applications that have a user data rate of greater than $20 \mathrm{kbps}$ at $100 \mathrm{~km} / \mathrm{hr}$ and $5 \mathrm{kbps}$ for fixed transmissions.

\section{3. $\quad$ Packet Error Rate \& Packet Loss}

Packet error Rate $P(E)$ is the percentage of packets containing at least one error, prior to applying error correction; packet loss $P(L)$ on the other hand, is the fraction of packets discarded due to uncorrected errors.

Both metrics i.e. $\mathrm{P}(\mathrm{E})$ and $\mathrm{P}(\mathrm{L})$, are closely related as will be shown; we start by capturing packet error, then use the result to discuss the packet loss.

\section{Packet Error}

The Packet error Rate $P(E)$, is related to the collision probability; this is explained as follows: When a collision occurs between the desired and the interfering packet, it is detected at the wireless receiver as signal to interference ratio (SIR), which is then mapped to bit error rate (BER) according to the modulation used [34].

Let $P(E F)$ be the probability of error free packet. Using the theorem of total probability, it can be expressed as follows :

$$
\begin{aligned}
& \mathrm{P}(\mathrm{EF})=\mathrm{P}(\mathrm{EF} \mid \mathrm{C}) * \mathrm{P}(\mathrm{C})+\mathrm{P}(\mathrm{EF} \mid \mathrm{NC}) *[1- \\
& \mathrm{P}(\mathrm{C})]
\end{aligned}
$$

Where $\mathrm{P}(\mathrm{EF} \mid \mathrm{C})$, is the probability of "error free packet", conditioned on the occurrence of a collision; $\mathrm{P}(\mathrm{EF} \mid \mathrm{NC})$ is the conditional probability of "error free packed" given no collision.

Let $B E R_{c}$ and $B E R_{n c}$ denote the bit error probability when there is a collision and no collision respectively; $\sim L$ is the number of bits involved in the impact. This is clearly a random variable that has a uniform distribution i.e. $U(0 ; M)$, where $M$ is $\min \left(N_{D} ; N_{l}\right) ; N_{D}$ and $N_{l}$ are the number of bits in desired and interfered packet respectively.

If we condition on $L=l$, we will get the following expressions:

$$
\left\{\begin{array}{ll}
P(E F \mid N C) & =\left(1-\mathrm{BER}_{n c}\right)^{N_{D}} \\
P(E F \mid C) & =\left(1-\mathrm{BER}_{c}\right)^{l}\left(1-B E R_{n c}\right)^{N_{D}-l}
\end{array}\right\}
$$

Now we can remove the condition on $L=1$, by averaging over all possible values:

$$
\left\{\begin{array}{l}
P(E F \mid N C)=\left(1-\mathrm{BER}_{n c}\right)^{N_{D}} \\
P(E F \mid C)=\frac{1}{N_{D}} \sum_{l=1}^{N_{D}}\left(\left(1-\mathrm{BER}_{c}\right)^{l}\left(1-B E R_{n c}\right)^{N_{D-l}}\right.
\end{array}\right\}
$$

By substituting (46) into (44), the packet error $P(E)=1-$ $P(E F)$ is obtained; the values for $B E R_{c}$ and $B E R_{n c}$ are calculated using formula (47), which provides the BER [39] for GFSK modulation at the bluetooth receiver. 
$B E R=Q_{1}(a, b)-\frac{1}{2} \exp \left(\frac{a^{2}+b^{2}}{2}\right) I_{0}(a, b)$

Where $Q_{1}($.$) is the first order Q$ function $I_{0}$ is the 0 -order modified Bessel function.

$$
\left\{\begin{array}{l}
a=\sqrt{\frac{r}{2}\left(1-\sqrt{\left.1-\left(\frac{\sin (2 * \pi * h)}{2 * \pi * h}\right)^{2}\right)}\right.} \\
b=\sqrt{\frac{r}{2}\left(1+\sqrt{\left.1-\left(\frac{\sin (2 * \pi * h)}{2 * \pi * h}\right)^{2}\right)}\right.}
\end{array}\right\}
$$

Where $\Gamma=\frac{E_{b}}{N_{0}}, \mathrm{E}_{\mathrm{b}}$ is the power for $\mathrm{BT}$ and $\mathrm{N}_{0}$ is the noise spectral density. Note that $\frac{E_{b}}{N_{0}}$ is replaced by SNR for $\mathrm{BER}_{n c}$ and by SINR in the case BER $\mathrm{B}_{\mathrm{c}}$ the modulation index $\mathrm{h}=0.32$

\section{Packet Loss}

Packets consist of three portion: the access code AC, the header(HE) and the payload (P); the AC and HE use error correction and if the operation fails, the packet is discarded;

$$
P(L)=1-\left(1-P_{A C}(L)\right)\left(1-P_{H E}(L)\right)\left(1-P_{p}(L)\right)
$$

therefore the probability of packet loss is approximately the probability of payload loss

$$
P_{p}(L) \text {, i.e. } P(L) \approx 1-\left(1-P_{P}(L)\right)=P_{P}(L)
$$

\section{CONCLUSION}

In order to quantify the performance of our process, both analytical performance as well as simulation studies were carried out. Different scenarios were investigated, with emphasis on dynamic channel environment. Our achievements were compared to the results of other adaptive hopping algorithms; it shows that SAFH outperforms the other schemes with respect to frame error rate, under static and dynamic sources of interferences. In addition it exhibits fast adjustment to changes in the environment and very stable operation i.e. less fluctuations.

\section{REFERENCES}

[1] Fanhua Shang a,n, L.C.Jiao a, JiarongShi a, FeiWang b, MaoguoGong a, "Fast Affinity Propagation Clustering: A Multilevel Approach", doi:10.1016/j.patcog.2011.04.032, Elsevier Pattern Recognition, 45, 474-486, (2012).

[2] Kiri Wagsta, Claire Cardie, Seth, Stefan Schroedl, "Constrained K-means Clustering with Background Knowledge", Proceedings of the Eighteenth International Conference on Machine Learning, $\mathrm{p}$. 577-584, (2001).

[3] Alan D. Amis, Ravi Prakash, Thai H.P. Vuong, Dung T. Huynh, "Max-Min D-Cluster Formation in Wireless Ad Hoc Networks", (2000).

[4] Seung-Hwan Lee, Yong-Hwan Lee, "Adaptive Frequency Hopping and Power Control Based on Spectrum Characteristic of Error Sources in Bluetooth Systems", Elsevier Computers and Electrical Engineering, 36, 341-351, doi:10.1016/j.compeleceng.2009.03.011, (2010).

[5] Hoi Kit Yip, Yu-Kwong Kwok, "Practical Channel State Aware And Cooperative Packet Scheduling Disciplines For Coordinating Colocated Bluetooth And IEEE 802.11b Devices", Elsevier Computer Communications, $\quad 30, \quad 1569-1587$, doi:10.1016/j.comcom.2007.01.010, (2007).

[6] Inn Inn Er, Winston K.G. Seah, "Performance Analysis of Mobility-Based D-Hop (Mobdhop) Clustering Algorithm For Mobile Ad Hoc Networks", Elsevier Computer Networks, 50, 33753399, doi:10.1016/j.comnet.2005.12.013, (2006).

[7] Sami Ben Cheikh, Tim Esemanny and Horst Hellbr"uck, "SAFH - Smooth Adaptive Frequency Hopping".

[8] Michael Cho-Hoi Chek and Yu-Kwong Kwok, "On Adaptive Frequency Hopping to Combat Coexistence Interference between Bluetooth and IEEE 802.11b with Practical Resource Constraints", Proceedings of the 7th International Symposium on Parallel Architectures, Algorithms and Networks (ISPAN'04), 1087-4089/04, IEEE, (2004).

[9] Michael Cho-Hoi Chek, Yu-Kwong Kwok, "Design And Evaluation of Practical Coexistence Management Schemes For Bluetooth And IEEE 802.11b Systems", Elsevier Computer Networks 51, 2086-2103， doi:10.1016/j.comnet.2006.10.009, (2007)

[10] Eric Lawrey, Cornelis Jan Kikkert, "Adaptive Frequency Hopping for Multiuser OFDM", Second International Conference on Information, Communications \& Signal Processing, ICICS'99, Singapore, 7-10 December, (1999).

[11] Jong-Woon Yoo, Member, IEEE, and Kyu Ho Park, "A Cooperative Clustering Protocol for Energy Saving of Mobile Devices with WLAN and Bluetooth Interfaces", IEEE Transactions on Mobile Computing, Vol. 10, No. 5, April (2011).

[12] Sasikanth Avancha, Anupam Joshi, Timothy Finin, "Enhancing the Bluetooth Service Discovery Protocol".

[13] Igor Sedov1, Stephan Preuß2, Clemens Cap3, Marc Haase1, Dirk Timmermann, "Time and Energy Efficient Service Discovery in Bluetooth". 
[14] M.Sughasiny and Dr.R.Dhanapal, "Performance Evaluation of An Anternative Controller For Bluetooth Service Discovery", ICTACT Journal on Communication Technology-Volume: 3, Issue: 2, pp. 529-534, (2012).

[15] M. Sughasiny and R. Dhanapal, "Performance Improvisation for Bluetooth Service Discovery Using Single Hop and Multi Hop Methods", ICTACT Journal On Communication Technology, June, Volume: 03, Issue: 02, Issn: 22296948(Online), (2012).

[16] Andrew Dursch, David C. Yen, Dong-Her Shih, "Bluetooth Technology: An Exploratory Study of The Analysis And Implementation Frameworks", Elsevier Computer Standards \& Interfaces 26, 263277, doi:10.1016/j.csi.2003.12.005, (2004).

[17] R. Mettala. Bluetooth Protocol Architecture. http://www.bluetooth.com/developer/whitepaper/, (1999).

[18] C.-F. Chiasserini, R.R. Rao, "Coexistence mechanisms for interference mitigation in the 2.4GHz ISM band", IEEE Transactions Wireless Communications 2 (5), 964-975, IEEE, (2003).

[19] G. Ennis, "Impact of Bluetooth on 802.11 Direct Sequences", IEEE 802.11-98/319, (1998).

[20] Y.-K. Kwok, Time-domain, frequency-domain, and network level resource management schemes in Bluetooth networks, in: Mihaela Cardei, Ionut Cardei, Ding-Zhu Du (Eds.), Resource Management in Wireless Networks, Kluwer Academic Publishers, (2004).

[21] Siemens, blue2net, <http://www.siemens.at/bluetooth/indexen.htm/>, (2004).

[22] B. Treister, H.B. Gan, K.C. Chen, H.K. Chen, A. Batra, and O.Eliezer, "Components of the AFH Mechanism", IEEE 802.15-01/252r0, <http://www.ieee802.org/15/pub/TG2-CoexistenceMechanisms.html/>, May, (2001).

[23] N. Golmie, R.E. Van Dyck, A. Soltanian, "Interference of Bluetooth and IEEE 802.11: Simulation Modeling And Performance Evaluation", in: Proc. 4th ACM International Workshop on Modeling, Analysis and Simulation of Wireless and Mobile Systems, pp. 11-18, (2001).
[24] G. Pei, M. Gerla, X. Hong, C.-C. Chiang, “A Wireless Hierarchical Routing Protocol with Group Mobility", in: Proc. IEEE WCNC '99, pp. 15381542, (1999).

[25] J. Linseed, "MEHTA: A Method for Coexistence between Co-located 802.11b and Bluetooth Systems", IEEE 802.15-00/360r0, <http:// www.ieee802.org/15/pub/TG2.html/>, Nov, (2000).

[26] G. Chinn et al., "Mobile PC platforms enabled with intel Centrino Mobile Technology", Intel Technology Journal 7 (2), (2003).

[27] D.P. Bovet, M. Cesati, "Understanding the Linux Kernel”, First ed., OR eilly and Associates, (2001).

[28] Z. Jiang, V. C. Leung, and V. W. Wong, "Reducing Collisions Between Bluetooth Piconets By Orthogonal Hop Set Partitioning", in Proc. IEEE Radio and Wireless Conference RAWCON, (2003).

[29] L. Stabellini, L. Shi, A. A. Rifai, J. Espino, and V. Magoula, "A new probabilistic approach for adaptive frequency hopping," in 20th IEEE International Symposium on Personal, Indoor and Mobile Radio Communications PIMRC., pp. 21472151, IEEE, (2009).

[30] S. Luca, "Toward Reliable Wireless Sensor Networks: Energy-Aware Distributed Interference Management for Unlicensed Bands", Ph.D. dissertation, KTH, Communication Systems, CoS, (2010).

[31] Q. Pang and V. C. Leung, "Improved channel classification and scheduling for non-collaborative bluetooth, WLAN coexistence," IEEE 63rd Vehicular Technology Conference, (2006).

[32] "WPANs specifications", [http://standards.ieee.org/about/get/802/802.15.html ], online; accessed 18-November, (2011).

[33] P. Popovski, H. Yomo, R. Prasad, and S. Member, "Dynamic adaptive frequency hopping for mutually interfering wireless personal area networks", ACM Mobihoc, vol. 4, pp. 991-1003, (2004).

[34] S. M., S. S., and G. D., "Coexistence of IEEE 802.11b and Bluetooth: an integrated performance analysis", Springer Science, (2008). 